\title{
PARTICIPAÇÃo NUM AMBIENTE DATIFICADo: QUESTÕES SOBRE LITERACIA DE DADOS
}

\author{
Miren Gutiérrez \\ Departamento de Estudos de Comunicação, Faculdade de Ciências \\ Sociais e Humanas, Universidade de Deusto, Espanha
}

\begin{abstract}
Resumo
No contexto político, entende-se por participação o envolvimento dos cidadãos na tomada de decisões, incluindo mecanismos para que as pessoas intervenham nas escolhas políticas e sociais, entre outras áreas de ação. Esses mecanismos são cruciais, pois a democracia depende da participação cívica na vida política. No entanto, na era do big data, a participação não é possível sem o acesso e controle de dados por parte das pessoas; isto é, os direitos civis tornam-se direitos digitais. Este artigo trata da literacia de dados como um filtro para a participação e do papel das pessoas comuns no ambiente e nos processos de datificação. Como a participação num mundo datificado depende da capacidade das pessoas de entrar na contenda, questões sobre onde se estabelecem as linhas de separação entre especialistas e não especialistas (ou seja, cidadãos comuns) e se a intervenção na infraestrutura de dados requer um grau de literacia de dados para participação efetiva constituem uma discussão relevante para a prática e teoria do ativismo como uma forma de envolvimento político ou cívico. O envolvimento político é entendido aqui como uma ação coordenada voltada para a resolução de problemas, campanhas e assistência aos cidadãos. Ou seja, para resgatar a participação política num domínio de dados, é necessário um certo grau de capacitação. Partindo de uma taxonomia do envolvimento em data mining (Kennedy, 2016) e casos empíricos de mapeamento de crises (Gutierrez, 2018a, 2018b), este artigo teórico propõe conceptualizações para pensar sobre as implicações da participação na contemporaneidade.
\end{abstract}

\section{PARTICIPATION IN A DATAFIED ENVIRONMENT: QUESTIONS ABOUT DATA LITERACY}

\begin{abstract}
In politics, participation can be understood as citizen involvement in decision making, including mechanisms for people to intervene in political and social choices, among other areas of action. Those mechanisms are crucial since democracy hinges on civic participation in political life. However, in the big data era, participation is not possible without people's access to and control of data; that is, civil rights become digital rights. This article deals with data literacy as a filter for participation in a datafied environment and the role of ordinary people in data processes. Because participation in a datafied world depends of people's ability to enter the fray, questions about where lines can be drawn to separate experts from non-experts (i.e. ordinary citizens) and whether intervention in the data infrastructure requires a degree of data literacy for effective participation constitute a relevant discussion for the practice and theory of activism as a form of political or civic engagement. Political engagement is understood here as coordinated action aimed
\end{abstract}


at problem-solving, campaigning and assisting others. Namely, to rescue political participation in a datafied domain, a degree of skill is necessary. Drawing from a taxonomy of data mining involvement (Kennedy, 2016) and empirical cases of crisis mapping (Gutierrez, 2018a; 2018b), this theoretical article offers conceptualisations to think about what participation entails today.

KEYWORDS

activism; datafication; data activism; ordinary people; participation; technopolitics

\section{INTRODUÇÃO}

Em política, a participação pode ser entendida como o envolvimento dos cidadãos na tomada de decisões, incluindo mecanismos para que as pessoas influenciem escolhas políticas e sociais, entre outras áreas de atuação (Conselho de Direitos Humanos, 2014). Existe um sentimento atual de frustração e deceção com a participação, capturado por diferentes autores em diversas situações, incluindo baixos níveis de envolvimento político entre os jovens na Grã-Bretanha (Fox, 2015), frustração participativa com processos institucionais em Espanha (Fernández-Martínez, García-Espín \& Jiménez-Sánchez, 2019) e frustração com o fraco desempenho do governo na Ásia (Sanborn, 2017), entre outros. No entanto, a participação ainda é considerada vital para a democracia, pois pode afetar os interesses e o bem-estar individuais e coletivos, e pode tornar as decisões mais informadas e legítimas. Os mecanismos que facilitam a participação na vida pública são cruciais, pois a democracia depende do envolvimento cívico na tomada de decisão política (Conselho da Europa, 2017). Embora não haja consenso sobre como defini-la, a democracia é baseada na igualdade de acesso, oportunidades e voz, bem como participação. Por exemplo, a revista liberal The Economist publica um Índice de Democracia todos os anos, que, além das liberdades políticas e eleitorais, leva em conta a participação dos cidadãos na vida política como um dos fatores fundamentais para determinar o nível de desenvolvimento democrático de um determinado país (Kekic, 2007). A participação importa; no entanto, a transformação das tecnologias de informação e comunicação (TIC) e a datificação - o processo de conversão de muitos aspetos de nossa vida em dados (Cukier \& Mayer-Schoenberger, 2013) - alteraram a participação, apresentando novos desafios e oportunidades para o envolvimento dos cidadãos na política.

As TIC têm sido celebradas como facilitadoras e criticadas como restritivas da participação política nos últimos anos. De fato, a participação cibernética (ou seja, participação política que tem lugar online) tem sido amplamente explorada por inúmeros autores e perspetivas, incluindo Alvarez e Hall (2008), Dahlberg e Siapera (2007), deSoto (2014), Mossberger, Tolbert e McNeal (2008), Milan (2013, 2015), Papacharissi (2019), Sampedro (2011), Thomas (2018) e Uldam e Vestergaard (2015), entre muitos outros. Estes autores fazem parte da academia dedicada ao estudo da tecnopolítica, ou o emprego diverso e conflituoso de TIC por governos, indivíduos, organizações civis e movimentos bottom-up. O seu trabalho envolve estudos sobre políticas "aprimoradas 
pela internet" (ou seja, e-government e política 2.0, que facilitam práticas existentes) e "a nova política habilitada pela internet", que se refere ao papel essencial que as TIC desempenham na organização do cidadão, participação, política contenciosa e processos deliberativos (Kurban, Peña-Lopez \& Haberer, 2016). No mesmo sentido, referindo-se à participação cibernética em processos eleitorais, Sampedro distingue entre cibernautas (ou seja, internautas tipicamente envolvidos em buscas online) e ciberativistas (isto é, ativistas envolvidos em petições, lobbying, fóruns, práticas deliberativas online e outros "usos tecnopolíticos" da internet) (Sampedro, 2011, p. 22). Ou seja, as TIC podem ser um obstáculo para a participação em torno de questões de acesso, ou podem não ter impacto sobre o acesso, mas também podem fortalecer formas predominantes de participação e apoiar novas formas de participação cidadã.

O conceito de comum é fundamental para considerar o papel capacitador ou incapacitante das TIC. Os estudos de ciência, tecnologia e sociedade (CTS) oferecem diferentes termos e descrições para caracterizar os tipos de práticas tecnológicas comuns. Dodge e Kitchin referem-se ao software como "objetos do quotidiano" fazendo cada vez mais a diferença na vida das pessoas (Dodge \& Kitchin, 2008). Referindo-se à Web 2.0 - conhecida como a web participativa ou um ambiente web que facilita a criação e a troca de conteúdo gerado pelo utilizador -, Beer (2009) fala sobre como o software classifica e mergulha em aspetos da nossa "vida quotidiana". Beer distingue três níveis de pesquisa relativos, em primeiro lugar, às "organizações que estabelecem e ativam aplicativos da Web 2.0"; segundo, as "infraestruturas de software e suas aplicações na web"; e terceiro, como os dois primeiros níveis "se desenvolvem na vida daqueles que usam (ou não usam) aplicações web específicas" (2009, p. 998). Este artigo baseia-se principalmente no terceiro nível de Beer, com foco no emprego quotidiano de tecnologia para participação.

Mais especificamente em conexão com o envolvimento cotidiano com a infraestrutura de dados - entendido como software, hardware e processos envolvidos na transformação de dados em valor -, Couldry e Powell discutem a atuação de pequenas "dinâmicas sociais"'. Esses agentes operam "com fins sociais além do objetivo básico de gerar e analisar dados (geralmente com fins lucrativos)" (Couldry \& Powell, 2014, p. 2). Desta forma, estes autores estabelecem uma diferença fundamental entre os empregos com e sem fins lucrativos no âmbito da infraestrutura de dados. Aqui o interesse é o emprego sem fins lucrativos de dados. Kennedy usa o termo comum para tipificar as práticas de data mining das redes sociais (Kennedy, 2016) inspiradas na determinação dos Estudos Culturais de reduzir as lentes académicas para captar níveis tangíveis de aplicações tecnológicas (McCarthy, 2008). À medida que os estudiosos ampliam as práticas comuns de data mining, "vemos os atores em organizações comuns baixando suas visões também, em termos de como imaginam que a data mining pode servir a seus propósitos" (Kennedy, 2016, p. 86). Ou seja, a observação das práticas de dados na base oferece uma visão das preocupações reais das pessoas comuns e como elas as abordam. O comum,

' Utiliza-se a expressão "dinâmicas" em vez de termos como "agentes" ou "atores", porque estes podem sugerir uma intervenção exclusivamente masculina. 
portanto, deve ser apreciado aqui como "o lugar comum, o aparentemente mundano" (Kennedy, 2016, p. 6), ao contrário do "extraordinário" (McCarthy, 2008).

Até ao momento, a ênfase nas estruturas e abordagens de cima para baixo à datificação implicou uma falta de atenção sobre a possibilidade de pessoas comuns atuarem com agência de dados (Kennedy, 2016), em termos comparativos. Em contraste, este artigo é focado em práticas de dados participativas de baixo para cima. A participação neste contexto serve como uma defesa contra os desequilíbrios de poder. Por exemplo, a primeira implantação da plataforma de mapeamento Ushahidi para dar voz e visualizar as vítimas do banho de sangue que se seguiu às eleições de 2007, no Quénia, conseguiu contornar o bloqueio de informações imposto pelo governo e pelos média (Gutierrez, 2018a). No entanto, discussões aprofundadas sobre o poder dos dados são evitadas aqui, já que o foco não é o empoderamento. Em vez disso, falo de assimetrias e intensidades na agência de dados, embora criem ou sejam consequência de diferentes distribuições do poder dos dados. Parafraseando Castells (2009), o poder dos dados deve ser entendido como a capacidade de impor interesses particulares aos processos de dados, colher os dados pessoais de outras pessoas e tomar decisões e colher benefícios daí resultantes.

A noção de práticas quotidianas de dados também atende ao propósito deste artigo por dois motivos. Primeiro, a ênfase aqui não é o cidadão comum per se, mas as suas práticas comuns na forma como lida com a infraestrutura de dados. Em segundo lugar, como mostra uma análise anterior (Gutierrez, 2018a), os cidadãos comuns raramente se envolvem sozinhos em atividades baseadas em dados "focadas na solução de problemas e ajudando os outros" (Zukin, Keeter, Andolina, Jenkins \& delli Carpini, 2006, p. 7). Os conjuntos de dados são muito complexos e grandes, e as causas sociais são complicadas demais para serem tratadas sem auxílio, de modo que esses agentes sociais geralmente organizam-se em grupos para colaborar em prol de uma causa comum (Gutierrez, 2018a). Embora a colaboração em projetos de dados seja um assunto relevante, discute-se agora se a agência de dados pode ser comum.

Outra questão fundamental para a participação é a ideia de igualdade; a esfera pública serve aqui como uma maneira de pensar sobre como a participação igualitária se manifesta. A esfera pública idealizada foi descrita como um espaço seguro em que os participantes se tratam uns aos outros como iguais para chegar a um entendimento mútuo; ou seja, na esfera pública habermasiana, todos são participantes (Habermas, 1991, pp. 33-34). Alguns autores observam a transição de uma esfera pública normativa para uma nova esfera pública em rede (Benkler, 2006; Quintanilha, 2018). Mas será que existe igualdade nessas novas esferas mediadas pela tecnologia? Hoje, os desafios na elaboração de processos de decisão participativa dentro das esferas públicas incluem a capacitação de diversos participantes para o exercício de agência similar. Bacon define participação implicitamente como uma atividade voluntária e aberta, que pode ser regulada por normas (Bacon, 2009, p. 235). Da mesma forma, essas novas esferas tecnológicas incorporam regras e filtros. Três exemplos demostram como funciona. No mapeamento de crises - ou o crowdsourcing e o mapeamento em tempo real dos dados 
dos cidadãos para o humanitarismo - qualquer um pode tornar-se humanitário digital desde que se registe, declare suas competências, contribuições potenciais para o mapa e depois cumpra as diretrizes para produzir informação verificada e fiável (Gutierrez, 2018c). Essas esferas são inclusivas, mas "estabelecem limites para a inclusão" (Gutierrez, 2018c). No caso do design do Ubuntu - o sistema operacional de código aberto e livre para computação na nuvem -, Bacon observa que "cada membro em potencial deve assinar o Código de Conduta do Ubuntu" antes de poder participar (Bacon, 2009, p. 235). E apesar da "lenta constituição e consolidação de uma nova esfera pública em rede" em Portugal, Quintanilha mostra também a apropriação por parte de muitas formas de participação pública no ciberespaço (Quintanilha, 2018). Ou seja, a participação nas esferas públicas de dados não é exatamente igual, mas é inclusiva, pois as pessoas ultrapassam cada vez mais o limiar para se sentar na mesa de negociações.

Este artigo analisa, dentro da estrutura teórica acima delineada - que é baseada na interseção entre estudos de ciência, tecnologia e sociedade e teoria da democracia -, de que modo a participação política será eventualmente possível num ambiente de dados. Em primeiro lugar, explora-se como a datilografia mudou a natureza da vida cívica e a maneira como as pessoas se envolvem em práticas participativas de dados; segundo, observa-se como o comum pode ser entendido nesse contexto; terceiro, com base na taxonomia de agentes de data mining (Kennedy, 2016), explora experiências de mapeamento de crises coletivas para oferecer uma classificação de papéis baseados em dados e sua intensidade participativa; finalmente, inspeciona os desafios de alfabetização de dados e as oportunidades de participação. A tecnologia alterou a forma como pensamos sobre participação e igualdade e gerou novas regras sobre quem pode ser um participante. Consequentemente, a dataficação introduz desafios específicos e oportunidades de participação, conforme explorado a seguir.

\section{Pessoas Como agentes de dados}

A datificação transformou a maneira como as pessoas participam da vida política. Primeiro, a própria natureza da vida cívica muda, uma vez que na época do big data, a participação real - do tipo a que Arnstein se refere quando fala sobre "o poder real de afetar o resultado do processo" (Arnstein, 1969, p. 216) - depende, pelo menos parcialmente, do acesso das pessoas ao digital (Sampedro, 2014, 2018). O big data emerge como um "espaço de ação variado, embora muito diferente dos espaços em que os atores sociais pré-digitais operavam" (Couldry \& Powell, 2014, p. 2). O surgimento da era do big data abriu as portas para novos tipos de intervenção cidadã, que poderiam ser divididos em duas categorias: a infraestrutura de dados como uma área de deliberação e contenção política e como uma ferramenta para a ação política. Por um lado, a vigilância de dados - a vigilância massiva de governos e corporações baseada em big data (van Dijck, 2014) - e a utilização da infraestrutura de dados para discriminar minorias e grupos vulneráveis [por exemplo, o uso do Facebook para estimular a limpeza étnica em Mianmar ou manipular as eleições de 2016, nos Estados Unidos (Whittaker et al., 2018)] teve a 
oposição de ativistas dos direitos civis. O big data como uma esfera de debate e campanha ganhou velocidade recentemente, especialmente em torno de questões de privacidade, justiça, governança e manipulação (Carlson, 2018; Naik, 2017) ${ }^{2}$. O movimento de dados abertos, por exemplo, redefiniu a democracia e a participação aplicando práticas da cultura open source à produção e uso de dados, alargando novas racionalidades em torno da informação que "pode apoiar a agência de públicos datificados" (Baack, 2015, p. 1). De um modo geral, o big data, enquanto debate, centra-se na privacidade de dados, bem como nos direitos de dados. Por outro lado, indivíduos e grupos estão a usar a infraestrutura de dados politicamente como uma ferramenta para resistir à coleta e manipulação maciça de dados, e para tomar decisões, gerando diagnósticos, soluções, contra-narrativas e mudanças sociais (Gutierrez, 2018a). Este artigo concentra-se no último tipo de intervenção cidadã, de acordo com a preferência de Kennedy de observar práticas pró-ativas de dados, em vez de se concentrar nas assimetrias, desafios e problemas apresentados pela infraestrutura de dados (Kennedy, 2016).

Em segundo lugar, a participação no novo ambiente exige mais do que apenas interesse em contribuir para a tomada de decisões; exige superar barreiras de participação. "Dados generalizados e as racionalidades quantitativas relacionadas criam novas pressões sobre os cidadãos comuns que desejam participar da vida cívica, social e cultural, à medida que se tornam mais orientados por dados" (Kennedy \& Hill, 2017, p. 2). Essas pressões incluem também obstáculos; por exemplo, é necessário um certo grau de especialização para a respetiva interpretação dos dados, para que eles não se convertam em informações úteis automaticamente. Por exemplo, o data mining é o processo de descobrir padrões em grandes conjuntos de dados usando algoritmos inteligentes, estatísticas e sistemas de bancos de dados (Association for Computing Machinery, 2006).

Embora Zukin et al. (2006, p. 7) refiram a necessidade de adquiri competências para a integração no envolvimento político, a participação num ambiente de dados requer capacidades específicas já que os obstáculos ao envolvimento com a infraestrutura de dados parecem evidentes. Por exemplo, Couldry e Powell observam que os processos de data mining, que podem levar a perceções sobre aspetos da vida cotidiana, não permitem que "esses insights sejam incluídos na experiência da vida cotidiana" (Couldry \& Powell, 2014, p. 4). Outro exemplo é o pleno emprego da API do Twitter - a interface de programação de aplicativos que permite o acesso aos dados; Puschmann e Burgess afirmam que, se um utilizador não entende como pode utilizá-la, não consegue interagir efetivamente com a plataforma (Puschmann \& Burgess, 2013, p. 11).

Devido a esses obstáculos, a contribuição das pessoas comuns na infraestrutura de dados é tipicamente limitada ao papel de produtores de dados inconscientes em esforços maciços de coleta e vigilância de dados, liderados por governos e corporações. $O$ comportamento quotidiano gera dados sem acarretar construção de significado ou mesmo consciência básica e consentimento dos geradores de dados, que, agregados, padronizados e analisados, produzem informações e valor para os colhedores. Este papel

${ }^{2}$ Informação também retirada de https://privacyinternational.org/long-read/2724/ every-police-force-uk-will-soon-use-body-worn-video-cameras-record-us-public 
passivo não é considerado na participação deste artigo, uma vez que não implica agência. Entende-se aqui agência, não como atos simples (por exemplo, clicar em um botão), mas como "os processos mais longos de ação baseados em reflexão, dando conta do que se fez, ainda mais basicamente, dando sentido ao mundo de modo a agir dentro de" (Couldry, 2013, p. 13). A participação de dados envolve um esforço de reflexão e superação de barreiras; não é algo que acontece espontaneamente. Ou seja, uma vez que o acesso (um pré-requisito) é permitido pelos tomadores de decisão ou alcançado pelos participantes, a participação real num ambiente de dados requer conhecimento e ação.

Apesar dos impedimentos, algumas pessoas estão a exercer sua agência de dados transformando dados em objetos do quotidiano. De facto, o emprego da infraestrutura de dados por pessoas para a solução de problemas e o envolvimento do cidadão pode ser considerado uma forma de tecnopolítica de baixo para cima. A ação baseada em dados pode tanto melhorar os tipos tradicionais de participação na política, quanto permitir novos tipos de participação. Um exemplo de dados que aprimora uma campanha política em andamento: a criação de uma plataforma que visualiza e mapeia incêndios florestais deliberados na Espanha, denominados España en Ilamas (Garcia Rey \& Garrido, 2016). Dois projetos, um na Indonésia (Radjawali \& Pye, 2015) e outro na região amazónica ${ }^{3}$, são exemplos do segundo tipo de participação. Esses projetos permitem cartografia radical (ou seja, mapas com funções novas e não convencionais), incorporando sistemas de alerta e gerando evidências, alertas e contra-narrativas em torno da propriedade da terra, recursos e política.

As novas práticas sociotécnicas de envolvimento com dados demonstram a possibilidade de agenciamento face à coleta massiva de dados por governos e corporações e podem ser observadas como expressões de ativismo de dados, ou a casualidade de dados e narrativas, baseadas em dados e táticas com ação coletiva e política. A disponibilidade de ferramentas para coletar e empregar dados de indivíduos e grupos impulsionou o surgimento do ativismo de dados (Milan \& Gutierrez, 2015), que inicialmente visava gerar ferramentas e áreas protegidas de comunicação para técnicos e ativistas contra a vigilância de dados. Mais recentemente, surgiu uma base proativa de envolvimento com dados e tecnologia, utilizando o potencial dos dados e das TIC para apoiar os cidadãos no exercício de sua agência democrática. As pessoas estão a envolver-se proativamente com a infraestrutura de dados para gerar dados nos seus próprios termos, fazer mapas alternativos, criar contra-narrativas e produzir soluções para seus problemas quotidianos, desafiando abordagens de cima para baixo (Gutierrez, 2018a). Nesse contexto, também são relevantes as discussões sobre a intensidade da participação. No caso da participação mediada por dados, a intensidade participativa é determinada não apenas pela abertura por parte dos decisores e pela motivação por parte dos participantes, mas também pelo nível de destreza em dados dos últimos, como veremos adiante.

Em suma, as práticas diárias de lidar com dados e com os resultados da análise de dados geram questões sobre participação. Por que é que a participação dos cidadãos nas práticas de dados é importante? De que maneira as pessoas são participantes desse

3 Ver http://rede.infoamazonia.org/ 
ambiente? Qual é a intensidade participativa suficiente ou possível em práticas comuns de dados? Porque o envolvimento político é importante como um amortecedor contra as assimetrias de poder, parece que os limiares que dividem os especialistas de não especialistas devem cair e que a intensidade participativa deve aumentar para resgatar a participação num ambiente de dados. O que se segue é uma análise da participação política na era do big data e as possíveis intensidades participativas, a partir da taxonomia de envolvimento de data mining de Kennedy (2016) e de casos empíricos de ativismo de dados de análises anteriores (Gutierrez, 2018a, 2018b), oferecendo conceções que podem servir como ferramentas heurísticas para pensar sobre o que a participação via ativismo de dados implica hoje.

\section{INTENSIDADES E ASSIMETRIAS}

Examinando o data mining, Kennedy distingue entre agência de trabalho, agência de utilizador e agência de tecnologia (Kennedy, 2016). Trabalhadores em processos de data mining - isto é, "engenheiros de software, cientistas de dados e outros trabalhadores" são indivíduos e organizações encarregados do trabalho invisível de produzir algoritmos (Kennedy, 2016, p. 57). Enquanto alguns autores referem algoritmos antropomorfizados (Kennedy, 2016, p. 57), dando a impressão de que agem por conta própria (Lash, 2007; Striphas, 2015), Kennedy fala sobre o papel significativo dos trabalhadores na formatação da vida social, através do exercício da sua agência algorítmica nos bastidores (Kennedy, 2016). Mas nem todos os trabalhadores estão na mesma posição na hierarquia. Kennedy cita Barocas e Selbst para notar que os trabalhadores de dados podem incluir tanto decisores quanto simples mineradores com diferentes responsabilidades e controle sobre os processos (Kennedy, 2016, p. 57). Como veremos adiante, o campo dos trabalhadores pode ser muito diversificado.

Os utilizadores, muitas vezes "concebidos como um grupo cujo trabalho (não remunerado) é explorado", são interessantes pelo seu "potencial de agência", segundo Kennedy (2016, p. 57). No sistema da plataforma dos média sociais - isto é, a "máquina comercial, orientada para o lucro, que explora os utilizadores mercantilizando os seus dados pessoais e o seu comportamento de uso" (Fuchs, 2011, p. 304) -, os utilizadores podem ser ativos na auto-promoção. Self-branders (utilizadores de plataformas) envolvem-se no "processo altamente autoconsciente de auto-exploração" para visibilidade e "ganho material ou status cultural" (Hearn, 2008, p. 204). Recorrendo à tradição da pesquisa de audiência, Kennedy observa que existem outras maneiras de pensar sobre os cidadãos, observando o que eles sentem sobre o seu uso de plataformas de média sociais. Os utilizadores podem censurar o conteúdo que produzem, motivados pela sua aspiração de equilibrar suas mensagens ou manipular os seus perfis para evitar a monitorização (Kennedy, 2016, p. 60). Ou seja, os utilizadores podem fazer mais do que apenas escolher um dispositivo, pagar a um provedor de serviços, clicar em um botão ou postar uma foto.

De facto, os cidadãos comuns podem atuar com agência de tecnologia (Kennedy, 2016); por isso, designo-os por agentes-tecno. As pessoas sempre se esforçaram para "se 
apropriar das tecnologias" e "adaptá-las aos significados que iluminam as suas vidas" (Feenberg, 1999, p.x). Ou seja, as pessoas normalmente transformam tecnologias em ferramentas que consideram úteis (Fischer, 1994, p. 25). Kennedy considera a tecno-agência reflexiva e localiza os casos empíricos que observa no campo da agência ética, ou agência que visa fazer o bem (Kennedy, 2016, p. 64). Reconhece as críticas geradas pelos usos da infraestrutura de dados para fins predatórios comerciais ou de espionagem, bem como as plataformas proprietárias de emprego, que incorporam assimetrias e lacunas, por organizações sem fins lucrativos (Kennedy, 2016). No entanto, Kennedy prefere concentrar-se em práticas comuns de data mining que constituem "uma contribuição positiva para a vida social" (Kennedy, 2016, p. 43). Esse tipo de envolvimento também é o foco aqui.

Indo além da data mining, para incluir o mapeamento participativo (ou seja, o emprego da infraestrutura de dados), integrei na Tabela 1 os três papéis que as pessoas adotam na maioria dos mapas de crise e as três funções de dados de Kennedy. Os mapas de crise são normalmente lançados em casos de emergência, localizando geograficamente dados de cidadãos voluntários para apoiar operações humanitárias quase em tempo real (Gutierrez, 2018b). Estes mapas dependem de humanitários digitais, que configuram a implantação usando diferentes plataformas de mapeamento de locais remotos (por exemplo, implantadores); agências humanitárias, que empregam as informações no local, e pessoas afetadas pelo desastre, também no local, que relatam dados através de diferentes canais (por exemplo, email, plataformas de média sociais, mensagens de texto) e usam as informações (por exemplo, repórteres). Os implantadores podem incluir trabalhadores assalariados de organizações humanitárias e especialistas voluntários que colaboram pro bono para lançar e gerir o mapa, um esforço que requer competências para adaptar a plataforma de mapeamento pronta a usar e o seu sistema de verificação, categorizar alertas e pedidos de assistência para que organizações humanitárias possam usá-los, traduzir as informações para e de idiomas locais, mapear locais desconhecidos, coordenar os voluntários e implementar uma estratégia de comunicação, entre outras tarefas (Gutierrez, 2018a). A Tabela 1 compara esses papéis com os trabalhadores, utilizadores e tecnoagentes de Kennedy a partir dos pontos de vista de sua intensidade e posição participativa na hierarquia.

\begin{tabular}{lcccc}
\hline & AsSALARIADOS & $\begin{array}{c}\text { ESPECIALISTAS/COM } \\
\text { COMPETÊNCIAS }\end{array}$ & INTENSIDADE & $\begin{array}{c}\text { PosiÇÃo NA } \\
\text { HIERARQUIA }\end{array}$ \\
\hline Trabalhadores & $\mathrm{Sim}$ & $\mathrm{Sim}$ & Alta & Topo \\
\hline Utilizadores & Não & Não & Baixa & Base \\
\hline Agentes-tecno & Não & $\mathrm{Sim}$ & Alta & Topo \\
\hline Implantadores do mapa & Alguns & $\mathrm{Sim}$ & Alta & Topo \\
\hline Repórteres de dados do mapa & Não & Não (mediados por dispositivos) & Média & Base \\
\hline Utilizadores do mapa & Não & Alguns & Média & Base \\
\hline
\end{tabular}


A intensidade participativa nas práticas de dados pode ser baixa (por exemplo, utilizadores de Kennedy), média (por exemplo, os repórteres do mapa contribuem voluntariamente com seus dados) ou alta (por exemplo, implantadores de mapa trabaIhando sem parar enquanto a crise se desdobra). Uma ideia que emerge desta comparação é que os principais cargos - sejam assalariados ou voluntários, independentes ou trabalhando dentro de uma organização - são caracterizados por altas intensidades participativas e um alto nível de especialização e investimento de tempo. Por exemplo, o mapeamento de crises geralmente envolve profissionais remunerados, encarregados pelas suas organizações de ajudar no esforço, trabalhando lado a lado com profissionais e especialistas que atuam pro bono. Ambos são competentes e dedicados ao ponto de alguns chegarem à exaustão no esforço de ajudar as vítimas (Gutierrez, 2018b).

As pessoas apanhadas num desastre vão além da descrição que Kennedy faz de um utilizador quando ele oferece os seus dados e informações para apoiar o esforço humanitário de forma proativa. A participação dos repórteres de dados no mapeamento de crise é voluntária e consciente, combinando com a definição de "agência" de Couldry (Couldry, 2013, p. 13). O seu acesso à tecnologia media a sua participação. Categorizei sua intensidade participativa como média, embora esses repórteres de dados às vezes invistam mais do que seu tempo e dados no apoio à operação humanitária, pois as suas localizações e identidades podem ser expostas em situações perigosas ou de conflito (Gutierrez, 2018b). Os repórteres dão um passo deliberado além do testemunho, cumprindo o que Schudson chama a sua obrigação "monitorial" de conhecer o suficiente para participar em assuntos políticos (1998). No mapeamento de crises, o emprego de dados dos cidadãos significou uma mudança de paradigma: não apenas os chamados não-especialistas são convocados para participar de emergências humanitárias juntamente com especialistas; novos agentes surgiram como resultado desse esforço (ou seja, os humanitários digitais ou os implantadores).

Assim, o comum não tem a ver com se os cidadãos são especialistas ou assalariados. Por exemplo, vítimas de desastres não são passivas ou ignorantes; pelo contrário, as evidências mostram que um fator significativo para a prontidão para desastres não é tecnologia ou meios logísticos, mas a experiência das pessoas que foram atingidas por uma catástrofe anteriormente e o seu conhecimento daí resultante ${ }^{4}$. O comum, então, deve ser associado a se as pessoas incorporaram práticas de dados cognitivas na vida quotidiana, como observado anteriormente.

\section{LITERACIA DE DADOS: BARREIRAS E OPORTUNIDADES}

No âmbito estrutural de oportunidades de participação, ainda há um número significativo de barreiras. Como argumentado anteriormente, as lógicas de dados atuais determinam cada vez mais a vida das pessoas, enquanto os "meios de participação dependem progressivamente da tecnologia, aumentando o risco de marginalizar as pessoas

\footnotetext{
4 Ver https://www.odi.org/our-work/disasters
} 
em contextos de desigualdade sociopolítica, cultural, económica e infraestrutural" (Wissenbach, 2019, p. 15). Um desafio na agência de dados, que aparece antes que muitos outros possam se materializar, é a "ansiedade dos números", que pode ser tão agudo que "a mera expectativa de fazer matemática" pode desencadear a rede de bloqueio do cérebro (Adelson, 2014). A ansiedade numérica ou matemática está relacionada com os dados. Kennedy observa que "abordar os requisitos de alfabetização de dados significa pensar sobre como aprendemos a relacionar-nos com números e estatísticas" (Kennedy, 2016, p. 235). A experiência negativa de dados e números de algumas pessoas pode tornar-se um obstáculo para a obtenção da agência de dados. Kennedy e Hill falam também sobre como as visualizações de dados podem gerar frustração, bem como sentimentos positivos (Kennedy \& Hill, 2017, p. 8). A ansiedade de números, matemática, dados e estatísticas parece ser um obstáculo para a participação num ambiente de dados quando se pensa em resgatar a participação.

Outras barreiras estão relacionadas à indústria de infraestrutura de dados e sua falta de representatividade (de género), o que também limita o envolvimento comum. $\mathrm{O}$ acesso a essas indústrias não é totalmente aberto, o que produz desigualdades nas próprias indústrias. Por exemplo, a indústria de machine learning emprega uma fração ainda menor de mulheres do que o resto do setor de tecnologia globalmente (Simonite, 2018), resultando em vieses de dados e algorítmicos (Wachter-Boettcher, 2017). A falta de representantes é outro desafio.

Mas quero concentrar-me aqui na literacia de dados, uma condição fundamental de possibilidade de participação, cuja ausência pode impor uma barreira formidável. Por exemplo, Turkoglu (2011, p. 141) ao discutir "literacia mediática crítica" e ao mesclar a tradição crítica da Escola de Frankfurt com abordagens de "literacia mediática" vê a literacia como pré-condição para a participação dos média. Na mesma linha, a literacia de dados poderia ser entendida como outra condição da possibilidade de participação num mundo de dados. Hoje, a agência de dados participativa depende de três fatores principais, todos relacionados com a literacia de dados: a) competências de dados (entendidas como competência nos processos que vão desde como os dados são coletados até à sua utilização); b) acesso a recursos e c) ocupar ou realizar regimes que permitam sua aplicação ordinária. Kennedy destaca dois desses fatores em relação às práticas de data mining:

tal como o data mining pode excluir populações dos seus cálculos algorítmicos devido às suas particularidades metodológicas, ela pode ser exclusiva também de outra forma, em termos de quem tem acesso a ferramentas e tecnologias de data mining e as competências necessárias para participar de operações orientadas por dados. (Kennedy, 2016, p. 64)

A distribuição do acesso aos dados e a capacidade de extrair valor deles é desigual, e isso leva a novas divisões digitais, que "destacam o caráter problemático e antidemocrático de tais desigualdades" (Kennedy, 2016, p. 53). Como os dados foram concedidos com certos poderes, influência e lógicas levantam questões políticas. Quem tem 
experiência e acesso à infraestrutura de dados decide como e quem gere os processos e o conhecimento resultante, o que, por sua vez, afeta o mundo social. Ruppert, Isin e Bigo (2017) colocam o surgimento de práticas como data science, data mining e data analysis como uma reconfiguração de poder e conhecimento. Sem entender "as condições de possibilidade dos dados", é difícil "intervir ou moldar a política de dados se por isso se entende a transformação de sujeitos de dados em cidadãos de dados" (Ruppert, Isin \& Bigo, 2017, p. 1). Concordando, Hintz, Dencik e Wahl-Jorgensen dizem que, se a cidadania hoje é baseada no uso ativo de dados e na participação, a sua legitimação requer uma compreensão das tecnologias, das estruturas e agentes que a tornam possível, bem como dos seus interesses, e de como eles podem ser usados em práticas comuns (Hintz, Dencik \& Wahl-Jorgensen, 2017, p. 735). Consequentemente, a literacia de dados envolvendo proficiência dos mesmos, bem como o acesso a ferramentas e a possibilidade de explorar a análise de dados, também precisa de ser abordada.

Quando as pessoas têm acesso a competências, meios e oportunidades, o ativismo de dados acontece. Vários exemplos mostram como estes três fatores trabalharam juntos no ativismo de dados. O primeiro é a apropriação e o uso de drones, concebidos originalmente para fins militares, como um método para produzir dados e contra-mapas que se opunham à "apropriação de terras" governamentais na Indonésia (Radjawali \& Pye, 2015). Radjawali e Pye afirmam que, para fazer esses mapas, os desafios passaram pelo aproveitamento das seguintes circunstâncias: a) as competências especializadas para operar os drones, produzir planeamento espacial e interpretar dados e imagens de satélite; b) os fundos para gerar fotografias aéreas de alta resolução capazes de capturar imagens claras (que foram disponibilizadas por doadores externos); e c) a relativa liberdade que permitiu que as comunidades que geriam os drones prestassem depoimento em tribunal contra as grandes corporações de data mining, em 2009 (Radjawali \& Pye, 2015, p. 3). Este exemplo também mostra que os dados não são o objetivo final neste tipo de ativismo; eles são uma ferramenta teleológica para atingir objetivos de campanha ou mobilização. O segundo exemplo é um estudo sobre o que faz um mapa mobilizar pessoas, mostrando que, além de recursos e ocasião, o emprego de conjuntos de dados ricos e complexos também é crucial; isto é, no ativismo de dados baseado em mapas, a credibilidade associada aos dados é essencial para incitar os seguidores a agir (Gutierrez, 2019a). Os casos examinados em Gutierrez (2019a) ilustram que mesmo o mapa ativista mais participativo depende do conhecimento tecnológico das pessoas que participam e colaboram no processo. Noutro estudo comprova-se que os esforços de dados - sejam eles ativistas ou não - geralmente dependem da participação dos cidadãos, pelo menos como repórteres de dados (Gutierrez, 2019b). Essas noções - as competências exigidas para o exercício da agência de dados e a participação de facto dos cidadãos em projetos de dados de todos os tipos - sugerem que os cidadãos comuns são muito mais engenhosos do que o previsto e confirma a ideia de que o comum se aplica se as práticas de dados se tornam correntes, e não se os cidadãos têm ou não conhecimento especializado. Além disso, os não especialistas podem tornar-se especialistas fazendo; isto é, participando cidadãos comuns adquirem nova consciência e poder 
(Baum, 2015). Gerar a terceira condição para a literacia de dados - a oportunidade -, parece então outro fator para resgatar a participação.

A literacia de dados poderia ser também empregada como uma perspetiva para analisar como as pessoas se envolvem com os dados, redirecionando a atenção da academia para as circunstâncias em que os utilizadores agem em "proprietary digitised environments" (Pybus, Cote \& Blanke, 2015, p. 4). Dada a disparidade entre aqueles que normalmente geram dados, pessoas e aqueles que ganham valor a partir de dados, corporações e governos, "é necessário abrir novas formas de literacia digital, tais como literacia de privacidade, literacia de informação, literacia de código, literacias algorítmicas, literacias de banco de dados e assim por diante" (Pybus et al., 2015, p. 4). Gray, Bounegru, Milan e Ciuccarelli (2016) falam sobre "literacia da infraestrutura de dados". Estas novas formas de literacia representam também áreas de oportunidade ativista. Com base em Baack (2015), essas áreas incluem um novo foco na abertura e no compartilhamento de dados, o que interromperia o monopólio de governos e empresas sobre dados; transferir o modelo de participação de código aberto - que é descentralizado, flexível, colaborativo, peer-to-peer e livre - para a participação política e um novo interesse nos mediadores como meios necessários para aceder a dados (por exemplo, jornalistas de dados e ativistas que abrem os seus conjuntos de dados para escrutínio público e livre). Sobre o último ponto, Hintz, Dencik e Wahl-Jorgensen observam que "a função de vigilância que tradicionalmente atribuímos ao jornalismo é crítica" (Hintz et al., 2017, p. 735). Baack sugere que as práticas e ideias do movimento de dados abertos são relevantes porque "ajudam a entender como a informação pode apoiar a agência de públicos e atores fora do grande governo e grandes negócios" (Baack, 2015, p. 1). A implicação é que, não apenas as pessoas devem integrar a literacia de dados no conjunto das suas competências democráticas, mas também que o ativismo e a colaboração com mediadores de dados são necessários para abrir espaços para a aplicação da literacia dados.

\section{DISCUSSÃo}

Este artigo termina com as perguntas iniciais. Como é que as pessoas comuns participam das práticas de dados? Qual é a intensidade participativa suficiente ou possível nas práticas comuns de dados? Em primeiro lugar, parece que, neste contexto, é mais interessante usar o termo comum em associação com a incorporação prática de dados nas vidas quotidianas das pessoas. Isto é, se o exercício da agência de dados se torna comum. Em segundo lugar, os cidadãos estão cada vez mais envolvidos com a infraestrutura de dados, atingindo as competências, os recursos e as oportunidades de explorá-la, transformando-a progressivamente num objeto comum. No entanto, para ocupar os altos cargos de decisão nas iniciativas de dados em todos os níveis (como trabalhadores, implantadores, utilizadores, repórteres e tecnoagentes), os cidadãos precisam de aumentar a intensidade do seu envolvimento com a infraestrutura de dados, o que também depende do seu nível de literacia de dados.

Por que é que a participação dos cidadãos na infraestrutura de dados é importante hoje em dia? A datificação de tudo apresenta um novo ambiente para participação 
política real, que requer agência cognitiva e literacia de dados como pontos de entrada, e resulta em novas práticas tecnopolíticas. O impedimento para a agência de dados pode ser intencional ou não. A participação hoje é ameaçada pela vigilância corporativa e governamental baseada em dados (Hintz et al., 2017, p. 732). A datificação fornece oportunidades massivamente aumentadas para entender, prever, abordar e manipular os cidadãos como indivíduos em tempo real (Tufekci, 2014). Estudos críticos alertaram sobre os perigos de deixar as decisões de dados ao mercado livre, às corporações ou até mesmo aos governos. Quer os impedimentos sejam intrínsecos à infraestrutura de dados, quer sejam impostos deliberadamente, fazem parte da "ambivalência" da tecnologia, que se refere, por um lado, a como é empregada para perpetuar hierarquias e garantir a continuidade do poder e, por outro lado, como uma ferramenta para minar essas mesmas hierarquias (Feenberg, 1999, p. 76). Tornar a infraestrutura de dados comum - uma questão chave para a democracia, porque a dataficação muda a natureza da vida cívica e aumenta os requisitos para participação - e aproveitar a sua ambivalência para "fazer o bem" (Kennedy, 2016, p. 71) requer mais do que o trabalho de um grupo de pesquisadores nos estudos críticos de dados; exige a participação ativa de pessoas comuns e organizações, promovendo oportunidades para criar os dados e para a sua utilização efetiva.

Para resgatar a participação e diminuir os patamares de entrada, questões como os dados e a ansiedade a eles associada devem ser abordadas; a literacia de dados como, por exemplo, o acesso a dados, meios e oportunidades - deve aumentar, e novos espaços colaborativos para legitimar a agência de dados devem ser criados para que a infraestrutura de dados se torne um objeto comum no envolvimento cívico. Pessoas e organizações já estão a trabalhar nesse sentido. Exemplos como o Medialab-Prado, em Madrid, que convida regularmente jornalistas, artistas, engenheiros e analistas de dados para trabalhar em conjunto para modelar projetos de dados, têm resultado em projetos em progressão, como o caso de España en llamas5; DataKind, que incentiva cientistas de dados a trabalhar pro bono com organizações sociais ${ }^{6}$; Data Science for Social Good, que treina cientistas de dados para lidar com problemas sociais, transferindo competências de dados no processo ${ }^{7}$ e Good Data, um projeto e um livro que expõe práticas éticas de dados, num processo de baixo para cima $^{8}$, revelam que as diferentes sensibilidades da sociedade podem utilizar os dados em torno do ativismo cívico.

Tradução: Ana Duarte Melo e Fábio Ribeiro

\footnotetext{
${ }^{5}$ Ver http://medialab-prado.es/article/que_es

${ }^{6}$ Ver http://www.datakind.org/about

${ }^{7}$ Ver http://dssg.io/projects/

${ }^{8}$ Ver http://networkcultures.org/blog/publication/tod-29-good-data/
} 


\section{NOTA DE TRADUÇÃo}

Tendo em conta que a versão original deste texto, em Inglês, apresenta diversos termos/neologismos cuja tradução para Português sugere diversas dificuldades, os tradutores entenderam manter o(a) conceito/expressão utilizado(a) de modo a representar a ideia inicial do modo mais aproximado possível.

\section{REFERÊNCIAS}

Adelson, R. (2014, 29 de agosto). Nervous about numbers. Retirado de www.psychologicalscience.org/ observer/nervous-about-numbers

Alvarez, R. M. \& Hall, T. E. (2008). Electronic elections: the perils and promises of digital democracy. Princeton: Princeton University Press.

Arnstein, S. R. (1969). A ladder of citizen participation. Journal of the American Planning Association, 35(4), 216-224.

Association for Computing Machinery (2006). Data mining curriculum: a proposal. Retirado de www.kdd.org/ curriculum/index.html

Baack, S. (2015). Datafication and empowerment: how the open data movement re-articulates notions of democracy, participation, and journalism. Big Data Q Society, 2(2), 1-11. https://doi. org/10.1177/2053951715594634

Bacon, J. (2009). The art of community: building the new age of participation (theory in practice). Sebastopol: O'Reilly Media, Inc.

Baum, H. S. (2015). Citizen participation. In J. D. Wright, Elsevier international encyclopedia of the Social $Q$ Behavioral Sciences (pp. 625-630). Londres: Elsevier.

Beer, D. (2009). Power through the algorithm? Participatory web cultures and the technological unconscious. New Media Q Society, 11(6), 985-1002. https://doi.org/10.1177/1461444809336551

Benkler, Y. (2006). The wealth of networks: how social production transforms markets and freedom. New Haven e Londres: Yale University Press.

Carlson, A. (2018, 8 de junho). Good data: challenging the colonial politics of knowledge. Retirado de https:// data-activism.net/2018/06/bigdatasur-blog-33-good-data-challenging-the-colonial-politics-of-knowledge/

Castells, M. (2009). Communication power. Oxford: Oxford University Press.

Conselho da Europa. (2017, 27 de setembro). Guidelines for civil participation in political decision making. Retirado de https://rm.coe.int/ guidelines-for-civil-participation-in-political-decision-making-en/16807626cf

Conselho de Direitos Humanos. (2014). Factors that impede equal political participation and steps to overcome those challenges. Retirado de https://www.ohchr.org/EN/HRBodies/HRC/RegularSessions/Session27/ Documents/A_HRC_27_29_ENG.doc

Couldry, N. (2013). A necessary disenchantment: myth, agency and injustice in a digital world. Retirado de http:// www.Ise.ac.uk/media@Ise/documents/MPP/Nick-Couldrys-LSE-INAUGURAL-SCRIPT.pdf

Couldry, N. \& Powell, A. (2014). Big Data from the bottom up. Big Data Q Society, 1(2), 1-5. https://doi. org/10.1177/2053951714539277 
Cukier, K. \& Mayer-Schoenberger, V. (2013). The rise of Big Data: how it's changing the way we think about the world. Foreign Affairs, 92(3), 28-40.

Dahlberg, L. \& Siapera, E. (2007). Radical democracy and the internet: interrogating theory and practice. Nova lorque: Palgrave Macmillan.

de Soto, P. (2014). Los mapas del \#15M: el arte de la cartografía multitud conectada. Retirado de https:// tecnopolitica.net/sites/default/files/pablodesoto.pdf

Dodge, M. \& Kitchin, R. (2008). Software, objects and home space. Retirado de https://personalpages. manchester.ac.uk/staff/m.dodge/Software_objects_and_home_space.pdf

Feenberg, A. (1999). Questioning technology. Londres e Nova lorque: Routledge.

Fernández-Martínez, J. L., García-Espín, P. \& Jiménez-Sánchez, M. (2019). Participatory frustration: the unintended cultural effect of local democratic innovations. Administration Q Society. https://doi. org/10.1177/0095399719833628

Fischer, C. S. (1994). America calling: a social history of the telephone to 1940. Berkeley: University of California Press.

Fox, S. (2015). Apathy, alienation and young people: the political engagement of British millennials. Retirado de http://eprints.nottingham.ac.uk/30532/1/Final\%20Corrected\%20Version\%20-\%20Apathy,\%20 Alienation\%2Oand\%20Young\%20People\%20The\%20Political\%20Engagement\%20of\%20British\%20 Millennials.pdf

Fuchs, C. (2011). Web 2.0, prosumption, and surveillance. Surveillance and Society, 8(3), 288-309. https://doi. org/10.24908/ss.v8i3.4165

Garcia Rey, M. \& Garrido, H. (2016). Datos sepultados bajo la ceniza (metodologia). Retirado de http:// espanaenllamas.es/datos-sepultados-bajo-la-ceniza-metodologia/

Gray, J., Bounegru, L., Milan, S. \& Ciuccarelli, P. (2016). Ways of seeing data: towards a critical literacy for data visualizations as research objects and research devices. In S. Kubitschko \& A. Kaun (Eds.), Innovative methods in Media and Communication Research (pp. 290-325). Londres: Palgrave Macmillan.

Gutierrez, M. (2018a). Data activism and social change. Londres: Palgrave Macmillan.

Gutierrez, M. (2018b). Maputopias: cartographies of knowledge, communication and action in the big data society - the cases of Ushahidi and InfoAmazonia. GeoJournal, 84(1), 101-120. https://doi.org/10.1007/ s10708-018-9853-8

Gutierrez, M. (2018c). The public sphere in light of data activism. Krisis, 1.

Gutierrez, M. (2019a). Cartographic emotions: how interactive maps mobilize people in geoactivism. In N. M. Engebretsen \& H. Kennedy (Eds.), Data Visualization in Society. Amesterdão: Universidade de Amesterdão.

Gutierrez, M. (2019b). The good, the bad and the beauty of "Good enough data". In A. Daly, K. Devitt \& M. Mann (Eds.), Good data: theory on demand, 29 (pp. 54-76). Amesterdão: Institute of Network Cultures. Retirado de http://ceem.unsw.edu.au/sites/default/files/documents/An\%20Energy\%20Data\%20 Manifesto\%20-\%20Chapter.pdf

Habermas, J. (1991). The structural transformation of the public sphere: an inquiry into a category of bourgeois society. Cambridge: The MIT Press. 
Hearn, A. (2008). "Meat, Mask, Burden": probing the contours of the branded "self". Journal of Consumer Culture, 8(2), 197-217. https://doi.org/10.1177/1469540508090086

Hintz, A., Dencik, L. \& Wahl-Jorgensen, K. (2017). Digital citizenship and surveillance society. International Journal of Communication, 11, 731-739.

Kekic, L. (2007). The Economist Intelligence Unit's index of democracy. Retirado de http://www.economist. com/media/pdf/DEMOCRACY_INDEX_2007_v3.pdf

Kennedy, H. (2016). Post, mine, repeat: social media data mining becomes ordinary. Londres: Palgrave Macmillan.

Kennedy, H. \& Hill, R. (2017). The feeling of numbers: emotions in everyday engagements with data and their visualisation. Sociology, 52(4), 830-848. https://doi.org/10.1177/0038038516674675

Kurban, C., Peña-Lopez, I. \& Haberer, M. (2016). What is technopolitics? A conceptual scheme for understanding politics in the digital age. In Atas da $12^{a}$ Conferência Internacional Internet, Law a Politics, Barcelona. Retirado de http://ictlogy.net/presentations/20160707_can_kurban_ismael_pena-lopez_ maria_haberer_-_what_is_technopolitics_conceptual_scheme.pdf

Lash, S. (2007). Power after hegemony: Cultural Studies in mutation? Theory, Culture Q Society, 24(55), 55-78. https://doi.org/10.1177/0263276407075956

McCarthy, A. (2008). From the ordinary to the concrete: Cultural Studies and the politics of scale. In M. White \& J. Schwoch (Eds.), Questions of method in Cultural Studies (pp. 21-53). Oxford: Wiley Blackwell.

Milan, S. (2013). Social movements and their technologies: wiring social change. Hampshire e Nova lorque: Palgrave Macmillan.

Milan, S. (2015). Liberated technology: inside emancipatory communication activism. In E. Gordon \& P. Mihailidis (Eds.), Civic media: technology, design, practice (pp. 107-124). Massachusetts: The MIT Press.

Milan, S. \& Gutierrez, M. (2015). Citizens' media meets Big Data: the emergence of data activism. Mediaciones, 14, 120-130.

Mossberger, K., Tolbert, C. J. \& McNeal, R. S. (2008). Digital citizenship: the internet, society, and participation. Cambridge: MIT Press.

Naik, R. (2017, 3 de outubro). Let's take back control of our data - it's too precious to leave to the tech giants. Retirado de https://www.theguardian.com/commentisfree/2017/oct/03/data-tech-giants-trail-digital-age

Papacharissi, Z. (2019). The virtual sphere: the internet as a public sphere. In M. Stempfhuber \& E. Wagner (Eds.), Praktiken der Überwachten (pp. 43-6o). Würzburg: Springer VS. https://link.springer.com/book/1 0.1007\%2F978-3-658-11719-1

Puschmann, C. \& Burgess, J. (2013). The politics of Twitter data. HIIG Discussion Paper Series, 2013-01. https:// doi.org/10.2139/ssrn.2206225

Pybus, J., Cote, M. \& Blanke, T. (2015). Hacking the social life of Big Data. Big Data \& Society, 2(2). https:// doi.org/10.1177/2053951715616649

Quintanilha, T. (2018). A contribution to the debate on the redefinition of the networked public sphere based on Portuguese public participation in cyberspace. Comunicação e Sociedade, 34, 287-304. https://doi. org/10.17231/comsoc.34(2018).2950 
Radjawali, I. \& Pye, O. (2015). Counter-mapping land grabs with community drones in Indonesia - land grabbing, conflict and agrarian-environmental transformations: perspectives from East and Southeast Asia. Comunicação apresentada na International Academic Conference, Chiang. Retirado de http://www.iss. nl/fileadmin/ASSETS/iss/Research_and_projects/Research_networks/LDPI/CMCP_8o-Radjawali_and_ Pye.pdf

Ruppert, E., Isin, E. \& Bigo, D. (2017). Data politics. Big Data Q Society, 4(2), 1-7. https://doi. org/10.1177/2053951717717749

Sampedro, V. (2011). Públicos y recursos tecnopolíticos: minorías insatisfechas y diques a la participación. In V. Blanco (Ed.), Cibercampaña: cauces y diques para la participación (pp. 11-39). Madrid: UCM Editorial Complutense.

Sampedro, V. (2014). El cuarto poder en red. Barcelona: Icaria.

Sampedro, V. (2018). Dietética digital: para adelgazar al gran hermano. Madrid: Icaria.

Sanborn, H. (2017). Broken back? Efficacy and participation in Asia's democracies. Asian Journal of Comparative Politics, 3(1), 13-24. https://doi.org/10.1177/2057891117699091

Schudson, M. (1998). Changing concepts of democracy. Comunicação apresentada na Conferência "Democracy and Digital Media", Massachusetts. Retirado de http://web.mit.edu/m-i-t/articles/schudson.html

Simonite, T. (2018, 17 de agosto). Al is the future - but where are the women? Retirado de https://www.wired. com/story/artificial-intelligence-researchers-gender-imbalance/

Striphas, T. (2015). Algorithmic culture. European Journal of Cultural Studies, 18(4-5), 395-412.

Thomas, S. (2018). Democratic deficits and digital compensation: the digital promises revisited. In T. Joseph \& S. Joseph (Eds.), Deliberative democracy. Understanding the Indian experience (pp. 142-159). Londres: Routledge. https://doi.org/10.4324/9780429486340

Tufekci, Z. (2014). Engineering the public: internet, surveillance and computational politics. First Monday, 19(7). https://doi.org/10.5210/fm.v19i7.4901

Turkoglu, N. (2011). Mediated public voices need theory to be heard. Management Communication Quarterly, 21, 141-158.

Uldam, J. \& Vestergaard (Eds.) (2015). Civic engagement and social media: political participation beyond protest. Londres: Palgrave Macmillan UK.

van Dijck, J. (2014). Datafication, dataism and dataveillance: Big Data between scientific paradigm and ideology. Surveillance \& Society, 12(2), 197-208.

Wachter-Boettcher, S. (2017). Technically wrong: sexist apps, biased algorithms and other threats of toxic tech. Nova lorque: Norton \& Company.

Wissenbach, K. (2019). Accounting for power in transnational civic tech activism: A communication-based analytical framework for media practice. The International Communication Gazette, Online First. https:// doi.org/10.1177/1748048519832779

Whittaker, M. et al. (2018). Al now report 2018. Retirado de https://ainowinstitute.org/Al_Now_2018_Report.pdf

Zukin, C., Keeter, S., Andolina, M., Jenkins, K. \& delli Carpini, M. X. (2006). A new engagement?: political participation, civic life, and the changing American citizen. Oxford: Oxford University Press. 


\section{NOTA BIOGRÁFICA}

Miren Gutiérrez é Doutorada em Comunicação. É Diretora do programa de Pós-Graduação "Análise de Dados, Pesquisa e Comunicação" da Universidade de Deusto, em Espanha. Investigadora no Overseas Development Institute (ODI), em Londres, e no projeto Datactive, na Universidade de Amsterdão, desenvolve pesquisa sobre ativismo de dados, nomeadamente a mudança social que é promovida pela utilização ativa dos dados digitais por parte de pessoas e organizações tendo em vista uma mudança social. Recentemente publicou o livro Data activism and social change, editado pela Palgrave-Macmillan.

ORCID: https://orcid.org/0000-0003-1527-3434

Email:m.gutierrez@deusto.es

Morada: Universidade de Deusto, Faculdade de Ciências Sociais e Humanas, Departamento de Estudos de Comunicação, Camino de Mundaiz 50, 20012 - San Sebastián, Espanha

* Submetido: 30/03/2019

* Aceite: 30/06/2019 